\title{
Three-dimensional Access Point Assignment in Hybrid VLC, mmWave and WiFi Wireless Access Networks
}

\author{
Charilaos C Zarakovitis ${ }^{*}$, Su Fong Chien $^{\dagger}$, Haris Pervaiz ${ }^{\S}$, Qiang Ni ${ }^{\S}$, John Cosmas $^{\ddagger}$, Nawar $_{*}$ Jawad $^{\ddagger}$, \\ Michail-Alexandros Kourtis , Harilaos Koumaras and Themistoklis Anagnostopoulos \\ * Media Networks Laboratory, Institute of Informatics and Telecommunications, NCSR "DEMOKRITOS", Athens, Greece, \\ \{c.zarakovits, akis.kourtis, koumaras, thmanagnostopoulos\}@iit.demokritos.gr \\ Advanced Informatics Lab, MIMOS Berhad, Kuala Lumpur, Malaysia, (sf.chien@mimos.my) \\ ${ }^{\S}$ InfoLab21, School of Computing and Communications, Lancaster University, Lancaster, United Kingdom, \\ \{c.zarakovitis, q.ni, h.b.pervaiz\}@ lancaster.ac.uk \\ Department of Electronic and Computer Engineering, Brunel University, London, United Kingdom, \\ \{john.cosmas, nawar.jawad\}@brunel.ac.uk
}

\begin{abstract}
To improve data speed and reliability, hybrid wireless networks combine two different Radio Access Technologies (RATs), such as Visible Light Communications (VLC), millimetre wave (mmWave), Wireless Fidelity (WiFi), 4G Long Term Evolution (LTE), etc. The Internet of Radio Light (IoRL) is a cutting-edge system paradigm to combine three RATs for taking advantage the vast VLC and mmWave spectrum with the ubiquitous coverage of $\mathrm{WiFi}$. In this respect, this work introduces a new convex optimisation-based solution method to optimise the three-dimensional (3D) Access Point Assignment (APÁ) problem of the IoRL system under individual user positioning, priority and minimum Quality-of-Service $(\mathrm{Q} O \mathrm{~S})$ constraints. We use both the IoRL real-world testbed and large-scale Maltab simulations to evaluate that our solution converges in linear time, and attains higher throughput-vs-fairness trade-off than existing efforts.
\end{abstract}

Index Terms - Access point assignment, hybrid network access, load balancing, optimisation analysis, software-defined network.

\section{INTRODUCTION}

To meet the future demand for mobile data traffic, wireless networks are becoming hybrid in nature by means of combining more than one Radio Access Technology (RAT). Within this direction, the Internet of Radio Light (IoRL) [1] stands as a cutting-edge hybrid network paradigm to combine the highspeed transmission of Visible Light Communications (VLC) and millimetre wave (mmWave) spectrum with the ubiquitous coverage of Wireless Fidelity (WiFi) sub-6GHz radio access. For such a network, the VLC and mmWave Access Points (APs) are to cover relatively small areas of approximately 2-3 meters diameter, while the WiFi is to access the, so-called, "dead zones", where the light and beams cannot spread.

In this respect, the IoRL system demonstrates that the synergy between VLC, mmWave and WiFi access networks can bring about significant performance improvements of indoor wireless communications services over each network working alone. The key idea is to associate users located into dead-zones to the WiFi APs and keep the rest of the users connected to the VLC and mmWave networks. Yet, the Access Point (AP) Assignment (APA) problem becomes challenging due to the completely different overlaying coverage of the coexisting networks, i.e., different types of APs differ in coverage range and system capacity. For example, when compared to VLC, a WiFi AP has a much larger coverage range (i.e. up to 40 meters indoor) but less network capacity (i.e. x10 lower throughput). On the other hand, the Quality-of-Service (QoS) requirements and priorities among users are heterogeneous in nature and depend on either single or various different parameters, like crediting, emergencies, body anatomies, etc. For example, humans are better to be prioritised to VLC APs, because they are radiation-free, rather than mmWave APs, Internet of Things (IoT) devices to WiFi, because it provides reliable and continuous access, rather than VLC, and so on. The important question is, therefore, how to distribute users among different types of APs for improving the overall performance of the hybrid network with an acceptable fairness level based on each user characteristics. Next Section reviews the most inclusive and recent attempts in the related literature and highlights the difficulties in tackling the APA problem.

\section{A. Related work}

The studies in [2]-[4] investigate the advantages in combining multiple VLC and WiFi APs, and conclude that by evaluating the Signal-to-Noise Ratio (SNR) of each channel to each user consists the most comprehensive APA criterion for migrating users to APs with higher throughputs. In this regard, [5] elaborates on SNR and user QoS criteria to formulate a centralised APA problem for maximising proportional fairness among users w.r.t AP assignments. The problem is proved mixedinteger non-linear programming (MINLP) with particularly high complexity, therefore, the same study, proposes a distributed version of the initial problem, which resolves in lower - yet exponential - complexity. Moreover, the APA scheme in [6] maximises system throughout considering that static users are connected to VLC APs, while moving users are associated with the WiFi AP. Using same scenario, [7] formulates a proportional fairness problem to tackle APA and radio resource allocation jointly, and another (second) problem to tackle APA and resource allocation separately. The study concludes that the joint problem can achieve better user QoS but with significantly greater complexity than resolving APA and resource allocation separately (x1000 higher). Furthermore, instead of assigning users to a specific AP, the work in [8] considers network hierarchical assignments by means of first assigning the network to each user, and then selecting the appropriate AP in the assigned network for each user. However, the problem in [8] draws on static system setting, which motivated [9], [10] and [11] to insight on dynamic system setup including channel blockage effects. The assumption is that users with high occurrence rate of channel blockages are assigned to the WiFi network, whereas users with low rate of blockages 


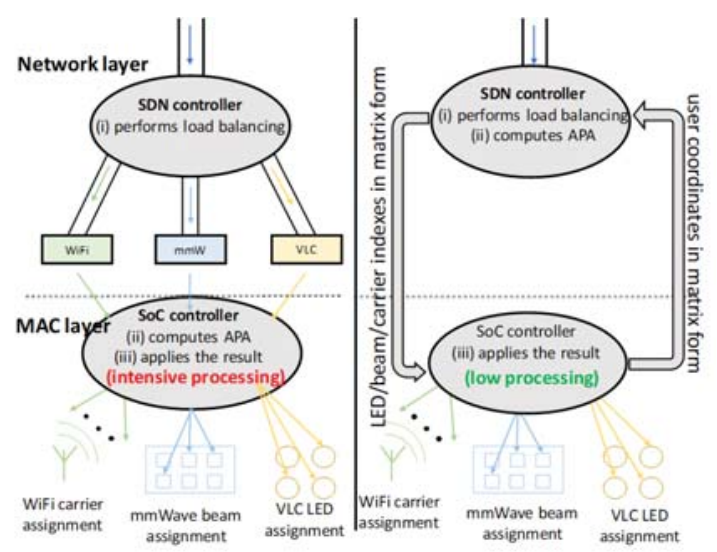

Fig. 1. High-level comparison between the conventional APA topology with intensive processing at the MAC layer (left), and the proposed SDN-driven approach with low processing at the MAC layer (right).

remain in the VLC network. Like in [5], these problems are also MINLP and resolve in high complexity. We emphasise that the solution methods used by the aforementioned studies [2]-[11] are either (i) optimisation-based algorithms, (ii) evolutional game theory, or (iii) fuzzy-logic algorithms. These methods are summarised and compared in [12], which concludes that fuzzy-logic algorithms outperform the other two methods when APA applies in static system setting, while optimisation-based algorithms are best for the dynamic systems by means of lower complexity and higher optimality level. Within this direction, [13] formulates a power and carrier allocation problem for energy efficiency optimisation in hybrid VLC and WiFi Software-Defined Network (SDN). It is shown that with the help of SDN, the APA strategy can be requested as an application from the Application layer, and then via the SDN controller, it can be coordinated and implemented as Medium Access Control (MAC) layer dynamic.

\section{B. Contributions and novelty}

The key contributions of this work are summarised as follows.

- Bearing the relevant works in [2]-[13] (and references within), we notice that the APA problem has been built onto a two-RAT system structure, i.e., either VLC-vsWiFi, VLC-vs-mmWave or mmWave-vs-WiFi. Instead, this work innovates by elaborating APA onto three-RAT hybrid network modelling, i.e., joint VLC-vs-mmWavevs-WiFi, which has yet been attempted, to the best of our knowledge. Under such consideration, the problem requires fundamentally different approach in how to be formulated and resolved because the determination space where the optimal AP assignment points can be determined is leveraged from two-dimensional (2D) to threedimensional (3D) space.

- In addition, given that by dimensioning up the APA problem structure naturally impacts the complexity of its solution in exponentially-like manner, we contribute by proposing a novel intelligent mathematical solution method, which bypasses the subgradient searching processes issued by conventional dual-Lagrangian optimisation and Dinckelbach-type algorithms [14], and resolves the 3D-APA problem in low (linear) complexity.

- Furthermore, we contribute by exploiting the abstraction between layers offered by SDN (i.e. a low-level layer can be used by any number of higher-level layers) to implement 3D-APA using the vast processing capacity of

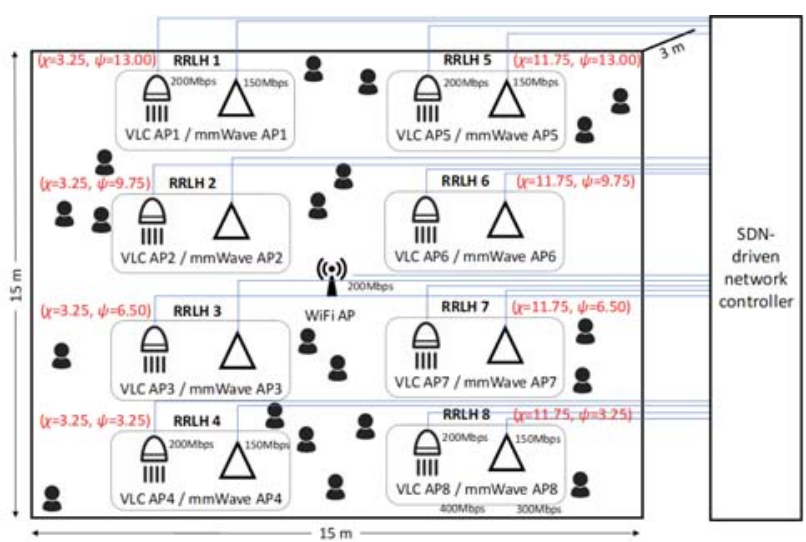

Fig. 2. Illustration of the hybrid VLC/mmWave/WiFi system model.

SDN controllers at Network layer, as illustrated in Fig 1. With such abstraction, the processing tasks of Systemon-Chip (SoC) controlling modules at the MAC layer are alleviated to straightforward apply the optimal APA result instead of running the whole algorithm, thus, the system becomes more effective to operate in real-time.

The remainder of this paper is organised as follows. Section II presents the system modelling, with Section III to formulate the 3D-APA optimisation problem. Section IV resolves the problem and highlights the implementation algorithm, with Section V to evaluate the outcomes through simulations. Finally, Section VI concludes the paper.

\section{SYSTEM MODELLING}

Let us consider a hybrid VLC, mmWave and WiFi access network, where multiple users are distributed into a $15 \times 15 \times 3$ meters open office space, as illustrated in Fig. 2. A single WiFi AP is placed on the ground at the center of the room, and eight Remote Radio Light Heads (RRLHs) are fitted on the ceiling to situate the LED photodiodes and the mmWave beam modules. Each light-beam and mmWave-beam acts as an individual high-speed AP covering a confined small area, while the WiFi AP provides lower-speed data rate but covers the entire room area. All APs are connected to the Network Gateway, which coordinates the data to the central Software Defined Network (SDN) controller that, in turn, performs the APA and load balancing processes among the three coexisting RATs, relying on SNR evaluations w.r.t user positioning in the room. For instance, assuming user $j=1, \ldots, J$ with $\left(\chi_{j}, \psi_{j}\right)$ coordinates and AP $i=1, \ldots, I$ with $\left(\chi_{i}, \psi_{i}\right)$ coordinates, the relative distance between the $i$ and $j$ points can be described by the straight line in the Euclidean space, i.e., $d_{i j}=\sqrt{\left(\chi_{i}-\chi_{j}\right)^{2}+\left(\psi_{i}-\psi_{j}\right)^{2}}$. Based on $d_{i j}$, the SNR function for each $(i, j)$ link between user $j$ and VLC AP can be represented as ${ }^{1}[15]$

$$
\mathrm{SNR}_{i j}^{\mathrm{VLC}}=\frac{I_{i j}}{N^{\mathrm{VLC}} \cdot \mathrm{BW}^{\mathrm{VLC}}}
$$

with $I_{i j}$ the sum of photons at AP $i$ converted into an electric current. Similarly, the SNR function for each $(n, j)$ link between user $j$ and mmWave AP as

$$
\begin{gathered}
\mathrm{SNR}_{n j}^{\mathrm{mmWave}}=10 \cdot \log _{10}\left|h_{n j}\right|^{2}+P_{n}+G_{j}-P L_{n j}^{\mathrm{mmWave}} \\
-\left(10 \cdot \log _{10} \mathrm{BW} \mathrm{W}^{\mathrm{mmWave}}+N^{\text {mmWave }}+N_{0}^{\mathrm{mmWave}}\right),
\end{gathered}
$$

${ }^{1}$ The detailed derivations of the VLC, mmWave and WiFi SNR functions are available in the IoRL deliverable 3.2 [15] and are omitted due to space limit. Besides, the main focus of this work is to introduce and evaluate the new solution method for the 3D-APA, instead of detailing the channel modellings. 
with $\left|h_{n j}\right|^{2}$ the normalised Gamma-random channel gain, $P L_{n j}^{\mathrm{mm} W a v e}$ the path-loss, $N_{0}^{\mathrm{mm} \text { Wave }}$ the noise figure, and the $\mathrm{SNR}$ function for each $(w, j)$ link between user $j$ and $\mathrm{WiFi}$ $\mathrm{AP}$ as

$$
\mathrm{SNR}_{w j}^{\mathrm{WiFi}}=\frac{G_{i j}^{\mathrm{WiFi}} \cdot P^{\mathrm{WiFi}}}{\mathrm{BW}^{\mathrm{WiFi}} \cdot N^{\mathrm{WiFi}}} .
$$

In (1), (2) and (3), $G^{(\cdot)}$ represents the channel gain, $P^{(\cdot)}$ the total transmit power, $\mathrm{BW}^{(\cdot)}$ the channel bandwidth, $N^{(\cdot)}$ the power spectral density of noise at the $i$-th VLC photodiode, $n$-th mmWave beam and $w$-th WiFi AP allocated to user $j$, respectively. We formulate next the $3 \mathrm{D}$-APA problem and highlight its respective LB scheme for improving the overall throughput of the hybrid network, namely, QoS-and-Priorityaware 3D-Access Point Assignment (QP3A).

\section{PROBLEM FORMULATION}

The QP3A problem draws on certain user criteria, like minimum user QoS requirements and user priorities, towards more practicable, fairer and less complex AP assignments than most related approaches, which use the empirical logic of Pure Opportunistic 2D-Access Point Assignment (PO2A). PO2A schemes are pure opportunistic because AP allocations are subject to certain throughout thresholds, such that, users with best positioning (or highest SNR) occupy most (if not all) APs, while the users in bad positioning, like in dead zones, are allocated with sufficiently less APs or even none. Due to unfairness among users, opportunistic scheduling is known to lead to resource scarcity and resource underutilisation [16].

To bypass such deficiencies, the proposed QP3A maximises the overall system throughput $R$, subject to a minimum QoS threshold for each user, i.e., $\mathrm{QoS}_{j}^{\mathrm{min}}$, and user priorities specified by the set $\left\{q_{j}^{(\varsigma)}\right\}$, with $\varsigma=1,2,3$ the Class index, i.e., $q_{j}^{\text {Class(1) }}>q_{j}^{\text {Class(2) }}>q_{j}^{\text {Class(3) }}$. This can be mathematically represented by the below optimisation problem.

$$
\begin{aligned}
& \max _{S_{\text {inwj }} \in\{0,1\}} R=\sum_{j=1}^{K} R_{j}^{\mathrm{VLC} / \mathrm{mm} \text { Wave/WiFi }} \\
& \text { to: }(\mathrm{C} 1) \sum_{j=1}^{K} q_{j}^{(\varsigma)}=1, \\
& \quad(\mathrm{C} 2) R_{j}^{\mathrm{VLC} / \mathrm{mm} W a v e / \mathrm{WiFi}} \geq \mathrm{QoS}_{j}^{\mathrm{min}}, \forall j, \\
& \quad(\mathrm{C} 3) \sum_{j=1}^{K} S_{\text {inw } j} \leq 1, \forall i, n, w,
\end{aligned}
$$$$
\text { subject to : (C1) } \sum_{j=1}^{K} q_{j}^{(\varsigma)}=1 \text {, }
$$

The utility function $R_{j}^{\mathrm{VLC} / \mathrm{mmWave} / \mathrm{WiFi}}$ in problem (4) represents the aggregated throughputs of each user over the VLC, mmWave, WiFi network access, i.e.,

$$
\begin{aligned}
R_{j}^{\mathrm{VLC} / \mathrm{mmWave} / \mathrm{WiFi}}=q_{j}^{(\varsigma)} \cdot \sum_{i=1}^{I} \sum_{n=1}^{N} \sum_{w=1}^{W} \hat{S}_{i n w j} \\
\times\left(R_{j}^{\mathrm{VLC}}+R_{j}^{\mathrm{mmWave}}+R_{j}^{\mathrm{WiFi}}\right) \\
=q_{j}^{(\varsigma)} \cdot \sum_{i=1}^{I} \sum_{n=1}^{N} \sum_{w=1}^{W} \hat{S}_{i n w j} \\
\times\left[\mathrm{BW}^{\mathrm{VLC}} \cdot \log _{2}\left(1+\mathrm{SNR}_{i n w j}^{\mathrm{VLC}}\right)\right. \\
+\mathrm{BW}^{\mathrm{mmWave}} \cdot \log _{2}\left(1+\mathrm{SNR}_{i n w j}^{\mathrm{mm} w a v e}\right) \\
\left.+\mathrm{BW}^{\mathrm{WiFi}} \cdot \log _{2}\left(1+\mathrm{SNR}_{i n w j}^{\mathrm{WiFi}}\right)\right],
\end{aligned}
$$

with $\hat{S}_{i n w j} \in\{0,1\}$ the element of the $3 \mathrm{D}$ matrix

$$
\begin{aligned}
& {\left[S_{I \times N \times W}\right]_{j}=\left[\hat{S}_{i n w j}\right]=\left[\hat{S}_{i n w}\right]_{j}^{T}=} \\
& \left.\left[\begin{array}{ccc}
\hat{S}_{111} & \cdots & \hat{S}_{1 n 1} \\
\vdots & \ddots & \vdots \\
\hat{S}_{i 11} & \cdots & \hat{S}_{i n 1}
\end{array}\right]\left[\begin{array}{ccc}
\hat{S}_{112} & \cdots & \hat{S}_{1 n 2} \\
\vdots & \ddots & \vdots \\
\hat{S}_{i 12} & \cdots & \hat{S}_{i n 2}
\end{array}\right]\left[\begin{array}{ccc}
\hat{S}_{11 w} & \cdots & \hat{S}_{1 n w} \\
\vdots & \ddots & \vdots \\
\hat{S}_{i 1 w} & \cdots & \hat{S}_{i n w}
\end{array}\right]\right]_{j}^{T}
\end{aligned}
$$

to denote the AP indexing of user $j$ over the VLC, mmWave and WiFi access w.r.t the SNRs given in (1), (2) and (3), respectively. Also, constraint $(\mathrm{C} 1)$ certifies the user priorities by setting the summation of all user weights equal to one, constraint (C2) specifies that each user is assigned with at least its minimum service requirement $\mathrm{QoS}_{j}^{\min }$, and (C3) is to ensure that each AP can be assigned to one user only.

\section{A. Problem relaxation}

The QP3A problem (4) is non-polynomial time (NP) hard and non-convex due to the district optimisation variable $S_{i n w j} \epsilon$ $\{0,1\}$. To make the solution of problem (4) tractable via convex optimisation analysis we relax the district variable $S_{i n w j}$ into continuous by setting $\overline{\operatorname{SNR}}_{i n w j}^{(\cdot)}=\operatorname{SNR}_{i n w j}^{(\cdot)} \cdot \hat{S}_{i n w j} \geq 0$, $\hat{S}_{\text {inwj }} \in[0,1]$, and

$$
\overline{\operatorname{SNR}}_{i n w j}^{(\cdot)}=\left\{\begin{array}{l}
\operatorname{SNR}_{i j}^{\mathrm{VLC}}, \text { for } w^{*}=n^{*}=0 \\
\operatorname{SNR}_{n j}^{m m W a v e}, \text { for } i^{*}=w^{*}=0 \\
\operatorname{SNR}_{w j}^{W i F i}, \text { for } i^{*}=n^{*}=0
\end{array}\right.
$$

which expresses the time-shared SNR that scales with $\hat{S}_{i n w j} \epsilon$ $[0,1][16]$. Using (7) and with the continuous variable $\hat{S}_{i n w j}$, the optimisation utility in (4) can be rewritten as

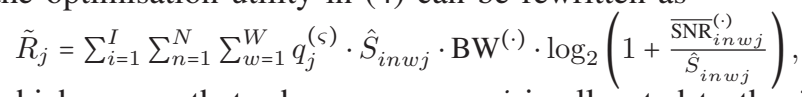

which means that when, e.g., user $j$ is allocated to the $i=2$ VLC AP, $n=7 \mathrm{mmWave}$ AP and not to the WiFi AP, then from (8) the user's time-shared throughout is given by

$$
\begin{aligned}
& \tilde{R}_{j}=0+\ldots+q_{j}^{(\varsigma)} \cdot \hat{S}_{200 j} \cdot \mathrm{BW}^{(\mathrm{VLC})} \cdot \log _{2}\left(1+\frac{\overline{\operatorname{SNR}}_{200 j}^{(\mathrm{VLC})}}{\hat{S}_{200 j}}\right)+\ldots \\
& +0+\ldots+q_{j}^{(\varsigma)} \cdot \hat{S}_{070 j} \cdot \mathrm{BW}^{(\mathrm{mmWave})} \cdot \log _{2}\left(1+\frac{\overline{\mathrm{SNR}}_{070 j}^{(\mathrm{mmWave})}}{\hat{S}_{070 j}}\right)+\ldots+0 .
\end{aligned}
$$

Based on $\tilde{R}_{j}$ in (8), we resolve next the problem (4) using standard-Lagrangian convex analysis, and present the pseudocode of the 3D-APA implementation algorithm.

\section{OPTIMAL 3D-APA SOLUTION, IMPLEMENTATION ALGORITHM AND COMPLEXITY}

The Lagrangian function of problem (4) is written as

$$
\begin{aligned}
L & =\sum_{j=1}^{K} \sum_{i=1}^{I} \sum_{n=1}^{N} \sum_{w=1}^{W} \hat{S}_{i n w j} \cdot \mathrm{BW}^{(\cdot)} \cdot \log _{2}\left(1+\frac{{\overline{\mathrm{SRR}_{i n w j}^{(\cdot)}}}_{\hat{S}_{i n w j}}^{(\cdot)}+}{}\right. \\
& +\mu\left(\sum_{j=1}^{K} q_{j}^{(\varsigma)}-1\right)-\sum_{i=1}^{I} \sum_{n=1}^{N} \sum_{w=1}^{W} \nu_{i n w} \cdot\left(\sum_{j=1}^{K} \hat{S}_{i n w j}-1\right) \\
& +\sum_{j=1}^{K} \xi_{j} \cdot\left(q_{j}^{(\varsigma)} \cdot \sum_{i=1}^{I} \sum_{n=1}^{N} \sum_{w=1}^{W} \hat{S}_{i n w j} \cdot \mathrm{BW}^{(\cdot)}\right. \\
& \left.\times \log _{2}\left(1+\frac{\overline{\mathrm{SNR}}_{\text {inwj }}^{(\cdot)}}{\hat{S}_{\text {inwj }}}\right)-\operatorname{QoS}_{j}^{\mathrm{min}}\right),
\end{aligned}
$$

with $\mu, \nu_{i n w}$ and $\xi_{j}$ the Lagrangian multipliers related to user priority constraint $(\mathrm{C} 1)$, minimum user QoS constraint $(\mathrm{C} 2)$ and AP allocation constraint (C3), respectively. The KarushKuhn-Tucker (KKT) optimality conditions yield $\frac{\partial L}{\partial \hat{S}_{i n w j}}=0$ [14], which, after some manipulations, resolves to the optimal 3D-APA indexing $\hat{S}_{i n w j}^{*}$, i.e.,

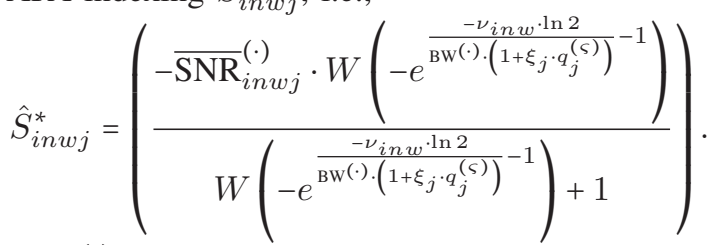

with $W(\cdot)$ the notation of the Lambert-W function [17]. However, $\hat{S}_{i n w j}^{*}$ in (10) is a function of multipliers $\nu_{i n w}$ and $\xi_{j}$ that are yet unknown. The traditional way to define these multipliers is to perform two separate searching processes for the subgradients of each possible $\nu_{i n w}^{*}$ and $\xi_{j}^{*}$ optimal point, as done in the relevant studies [2]-[11] and [13]. Yet, recalling that the determination space of $\hat{S}_{i n w j}^{*}$ is threedimensional for each user $j$, such seeking processes are likely to explode the algorithm's complexity in the exponential order 
of $\mathcal{O}\left(K^{I \times N \times W}\right)$, with $\mathcal{O}(\cdot)$ the big-O notation. Additionally, the processes may not always converge because an optimal point can have more than one subgradients or even none [14].

\section{A. Proposed convex optimisation-based solution method}

To resolve the QP3A problem in low linear complexity and guarantee its convergence, we propose a new solution method ${ }^{2}$ that embraces the Lambert-W function properties [17]. Particularly, we define $z\left(\nu_{i n w}, \xi_{j}, q_{j}^{(\varsigma)}\right)=-e^{\frac{-\nu_{i n w} \cdot \ln 2}{\mathrm{BW}(\cdot) \cdot\left(1+\xi_{j} \cdot q_{j}^{(\varsigma)}\right)}}$, such that $\left(1+\frac{\overline{\operatorname{SNR}}_{i n w j}^{(\cdot)}}{\hat{S}_{i n w j}}\right)=-\frac{1}{W(z)}$, and $W(z) \cdot e^{W(z)}=z$, which rewrites the Lagrangian function in (9) as

$$
\begin{aligned}
L & =\sum_{j=1}^{K} \sum_{i=1}^{I} \sum_{n=1}^{N} \sum_{w=1}^{W}\left[\frac{-\overline{\operatorname{SNR}}_{i n w j}^{(\cdot)} \cdot W(z) \cdot \mathrm{BW}^{(\cdot)} \cdot\left(1+\xi_{j} \cdot q_{j}^{(\varsigma)}\right)}{W(z)+1}\right. \\
& \left.\times \log _{2}\left(\frac{-1}{W(z)}\right)\right]-\sum_{j=1}^{K} \xi_{j} \cdot Q o S_{j}^{\min }+\mu\left(\sum_{j=1}^{K} q_{j}^{(\varsigma)}-1\right) \\
& -\sum_{i=1}^{I} \sum_{n=1}^{N} \sum_{w=1}^{W} \nu_{i n w} \cdot\left(\sum_{j=1}^{K}\left(\frac{-\overline{\operatorname{SNR}}_{i n w j}^{(\cdot)} \cdot W(z)}{W(z)+1}\right)-1\right) .
\end{aligned}
$$

In view of (11) and from the KKT conditions we calculate

$$
\begin{aligned}
& \frac{\partial L}{\partial \nu_{i n w}}=0 \Rightarrow \sum_{j=1}^{K}\left(\frac{{\overline{\mathrm{SNR}_{i n w j}}}_{(\cdot)} \cdot W(z)}{(W(z)+1)^{2}}\right) \cdot(W(z)+3)+1=0
\end{aligned}
$$

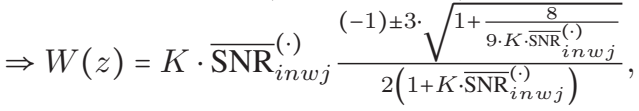

$$
\begin{aligned}
& \frac{\partial L}{\partial \xi_{j}}=0 \Rightarrow \sum_{i=1}^{I} \sum_{n=1}^{N} \sum_{w=1}^{W}\left[\frac{-\overline{\operatorname{SNR}}_{i n w j \cdot}^{(\cdot)} \cdot W(z)}{(W(z)+1)^{2}}\right. \\
& \times \frac{\mathrm{BW}^{(\cdot)}}{\ln 2} \cdot q_{j} \cdot(W(z)+1)^{2}+\frac{\ln 2}{\mathrm{BW}^{(\cdot)}} \frac{\nu_{i n w}}{\left(1+\xi_{j} \cdot q_{j}\right)} \\
& \times(W(z)+1)-\left(\frac{2 \cdot \ln 2}{\mathrm{BW}^{(\cdot)}} \cdot \frac{\nu_{i n w}}{\left(1+\xi_{j} \cdot q_{j}^{(\mathrm{s})}\right)}\right)-Q o S_{j}^{\min }=0 .
\end{aligned}
$$

By substituting (12) into (13), we obtain the optimal 3D-APA index $\tilde{S}_{i n w j}^{*}$ independent from the multipliers $\nu_{i n w}$ and $\xi_{j}$, as

$$
\tilde{S}_{i n w j}^{*}=\left(\frac{\frac{2}{K}-3 \cdot \overline{\mathrm{SNR}}_{i n w j}^{(\cdot)} \cdot\left(\sqrt{1+\frac{8}{9 \cdot K \cdot \mathrm{SNR}_{i n w j}^{(\cdot)}}}-1\right)}{\left(3 \cdot \sqrt{1+\frac{8}{9 \cdot K \cdot \overline{\mathrm{SNR}}(\cdot)}-1}\right)}\right) .
$$

However, due to the lack of multipliers, the optimal $\tilde{S}_{i n w j}^{*}$ in (14) is neither dependent on $\mathrm{QoS}_{j}^{\min }$ nor on $q_{j}^{(\varsigma)}$, meaning that it does not ensure that constraints (C1)-(C3) can be always satisfied. Therefore, we need to derive an $\tilde{S}_{i n w j}^{*}$-vs-QoS ${ }_{j}^{\mathrm{min}}$ vs- $q_{j}^{(\varsigma)}$ criterion to guarantee the feasibility of these constraints. We do so, by recalling the KKT of (11) w.r.t $\nu_{i n w}$, i.e.,

$$
\begin{aligned}
& \frac{\partial L}{\partial \nu_{i n w}}=0 \Rightarrow \ldots \Rightarrow z=W(z) \cdot e^{W(z)}=\tilde{S}_{i n w j}^{*} \cdot e^{\tilde{S}_{i n w j}^{*}} \\
& \Rightarrow{ }^{\xi_{j}=q_{j}, \mu=-Q o S_{j}^{\mathrm{min}}} \Rightarrow \nu_{i n w}=-\frac{\mathrm{BW}^{(\cdot)}}{\ln 2} \cdot\left(\ln \left(-\tilde{S}_{i n w j}^{*}\right)\right. \\
& \left.+\tilde{S}_{i n w j}^{*}+1\right) \cdot\left(1+\left(q_{j}^{(\varsigma)}\right)^{2}\right) \text {. }
\end{aligned}
$$

By substituting (15) into (13) and resolving w.r.t $\frac{Q o S_{j}^{\min }}{q_{j}^{(\varsigma)}}$, the criterion to guarantee that the priority constraint $(\mathrm{C} 1)$ and the QoS constraint $(\mathrm{C} 2)$ can be always satisfied is derived as

\footnotetext{
${ }^{2}$ The proposed solution method involves extended analysis with various combinations among theorems, which are difficult to include in this paper. Therefore, we present the key conclusions and derivations of our analysis, so as, the reader can realise the rationale and flow of the new method.
}

$$
\begin{aligned}
& \frac{Q o S_{j}^{\mathrm{min}}}{q_{j}^{(\varsigma)}} \leq \sum_{i=1}^{I} \sum_{n=1}^{N} \sum_{w=1}^{W}\left[2-3 \cdot K \cdot \overline{\mathrm{SNR}}_{i n w j}^{(\cdot)}\right. \\
& \times\left(-1+\sqrt{1+\frac{8}{9 \cdot K \cdot \overline{\mathrm{SNR}}_{i n w j}^{(\cdot)}}}\right) \\
& \quad \times\left(\left(-\frac{\mathrm{BW}^{(\cdot)}}{\ln 2}\right)\left(\ln \left(-\tilde{S}_{i n w j}^{*}\right)+\tilde{S}_{i n w j}^{*}+1\right)\right) \\
& \left.\quad \times \frac{\left.\left(K \cdot \overline{\mathrm{SNR}}_{i n w j}^{(\cdot)}\left(-5+3 \cdot \sqrt{1+\frac{8}{9 \cdot K \cdot \overline{\mathrm{SNR}}_{i n w j}^{(\cdot)}}}\right)-4\right)\right]}{K^{2} \cdot\left((-1)+3 \cdot \sqrt{1+\frac{8}{9 \cdot K \cdot \overline{\mathrm{SR}}_{i n w j}^{(\cdot)}}}\right)^{2}}\right] \\
& +\left[\frac{\mathrm{BW}^{(\cdot)}}{\ln 2} \cdot \frac{\overline{\mathrm{SNR}}_{i n w j}^{(\cdot)}}{2\left(1+K \cdot \overline{\mathrm{SNR}}_{i n w j}^{(\cdot)}\right)}\right]
\end{aligned}
$$

which combines $\tilde{S}_{i n w j}^{*}, \operatorname{QoS}_{j}^{\text {min }}$ and $q_{j}^{(\varsigma)}$ into a single function. The 3D-APA QP3A algorithm follows readily from (16). The order of complexity of the proposed Algorithm 1 is due

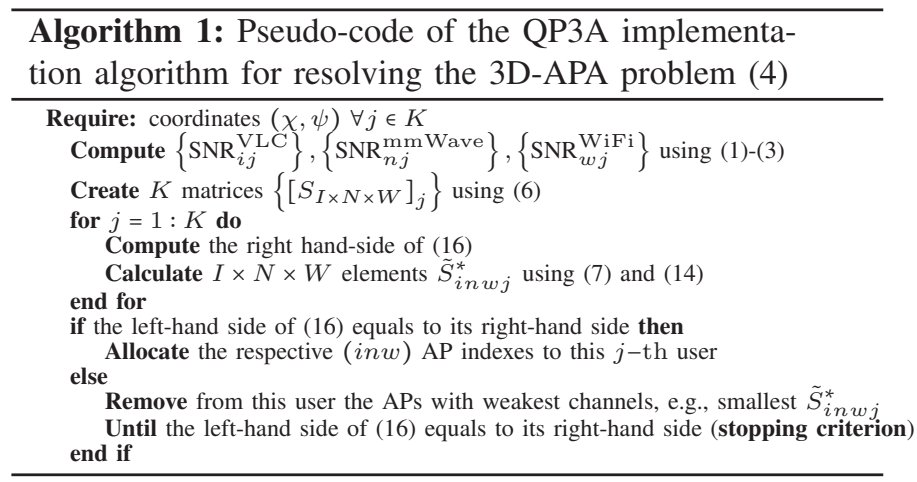

to the seeking process in the number of system users, which is linear, i.e., $\mathcal{O}(K \times I \times N \times W)$ and significantly less than the order of complexity of PO2A, i.e., $\mathcal{O}\left(K^{I \times N \times W}\right)$. Next Section evaluates the theoretical outcomes via the real-world IoRL SDN testbed as well as through Matlab simulations.

\section{EVALUATION MODELLING AND SIMULATION RESULTS}

For our evaluations we consider the hybrid network topology illustrated in previous Fig. 2, where the 8 RRLHs provide access to $K=20$ users that are uniformly distributed between 0 and 2 meters within the $15 \times 15 \times 3$ meters squared office and have different priorities and minimum QoS requirements as specified in Table I. For the fair comparisons between the proposed QP3A and the conventional PO2A (e.g. recall Section III for details), we consider that the VLC, mmWave and WiFi channel setting is similar to the studies in [2]-[4], [9]-[11] and [13], which, by considering the user coordinates in Table I, results to the normalised channel gains in Table $\mathrm{II}^{3}$.

\section{A. Evaluations using the IoRL real-world SDN testbed}

We instantiate both the PO2A and QP3A algorithms in the form of Virtual Network Functions (VNFs) onboarded as .qcow2 image files at the OpenStack repository of the IoRL SDN Dell R730xd server. The management of the LED photodiodes with the mmWave beam modules (situated within the eight RRLHs) and the WiFi module (placed at the center of the room) is driven by a Ryu controller [18], which installs, updates and deletes SDN rules respective to the APA

\footnotetext{
${ }^{3}$ The WiFi radio carriers are 4096 in total and cannot be included in Table II. Yet, we report that under the considered evaluation modelling, the WiFi channel gains vary between -11 and $-13 \mathrm{dBm}$.
} 
TABLE I

INDIVIDUAL USER PRIORITIES, MINIMUM QOS REQUIREMENTS AND INDICATIVE POSITIONING COORDINATES AT RANDOM TIME INSTANCE

\begin{tabular}{|c|c|c|c|}
\hline $\begin{array}{l}\text { User } \\
\text { index } \\
j\end{array}$ & $\begin{array}{l}\text { User priority } \\
q_{j}^{(\varsigma)}\end{array}$ & $\begin{array}{l}\text { User QoS } \\
\text { requirement } \\
\mathrm{QoS}_{j}^{\text {min }}\end{array}$ & $\begin{array}{l}\text { User coordinates }\left(\chi_{j}, \psi_{j}\right) \\
\text { at random time instance } \\
\text { in meters }\end{array}$ \\
\hline 1 & 0.02 (Class 3) & $50 \mathrm{Mbps}$ & $1.7882 \quad 11.5797$ \\
\hline 2 & 0.02 (Class 3) & $50 \mathrm{Mbps}$ & 14.097413 .9928 \\
\hline 3 & 0.02 (Class 3) & $50 \mathrm{Mbps}$ & $\begin{array}{ll}9.6833 & 14.5911 \\
\end{array}$ \\
\hline 4 & 0.02 (Class 3) & $50 \mathrm{Mbps}$ & $7.1919 \quad 2.8804$ \\
\hline 5 & 0.02 (Class 3) & $50 \mathrm{Mbps}$ & 9.5898 \\
\hline 6 & 0.02 (Class 3) & $50 \mathrm{Mbps}$ & $8.1707 \quad 10.4440$ \\
\hline 7 & 0.02 (Class 3) & $50 \mathrm{Mbps}$ & 9.7097 \\
\hline 8 & 0.055 (Class 2) & $100 \mathrm{Mbps}$ & $8.1583 \quad 7.8811$ \\
\hline 9 & 0.055 (Class 2) & $100 \mathrm{Mbps}$ & $10.8157 \quad 7.9552$ \\
\hline 10 & 0.055 (Class 2) & $100 \mathrm{Mbps}$ & $\begin{array}{ll}7.8374 & 12.9171 \\
\end{array}$ \\
\hline 11 & 0.055 (Class 2) & $100 \mathrm{Mbps}$ & $14.9056 \quad 7.2728$ \\
\hline 12 & 0.055 (Class 2) & $100 \mathrm{Mbps}$ & $3.2801 \quad 5.9018$ \\
\hline 13 & 0.055 (Class 2) & $100 \mathrm{Mbps}$ & $1.5870 \quad 10.0715$ \\
\hline 14 & 0.055 (Class 2) & $100 \mathrm{Mbps}$ & $1.6455 \quad 11.1189$ \\
\hline 15 & 0.079 (Class 1) & $150 \mathrm{Mbps}$ & 0.9539 \\
\hline 16 & 0.079 (Class 1) & $150 \mathrm{Mbps}$ & 6.0687 \\
\hline 17 & 0.079 (Class 1) & $150 \mathrm{Mbps}$ & 6.7256 \\
\hline 18 & 0.079 (Class 1) & $150 \mathrm{Mbps}$ & 11.45263 .9322 \\
\hline 19 & 0.079 (Class 1) & $150 \mathrm{Mbps}$ & $5.4872 \quad 8.7914$ \\
\hline 20 & 0.079 (Class 1) & $150 \mathrm{Mbps}$ & 0.6668 \\
\hline
\end{tabular}

strategy of each algorithm. Ryu uses (i) the OpenFlow version v.1.3. protocol for communicating with the RRLHs and WiFi, along with (ii) Mininet simulator and (iii) iperf for building the network virtualisation infrastructure and performing measurements, respectively. As observed by the outcomes in Table 4, PO2A allocates the AP with the maximum channel gain corresponded to each user, while the AP allocations in QP3A follow a fairer rational according to users' Classes. For example, in PO2A the Class 1 user 20 (i.e. highest-priority and $Q o S_{20}^{\min }=150 \mathrm{Mbps}$ ) and the Class 2 users 12 and 10 (i.e. medium priority and $Q o S_{20}^{\mathrm{min}}=100 \mathrm{Mbps}$ ) are assigned to the WiFi network meaning that their minimum QoS is not fulfilled. In contrast, all low-priority Class 3 users (except user 3) attain VLC or mmWave access, which however is suboptimal as these users occupy high-speed APs that could be used for serving the highly-demanded users 11, 12 and 20. On the other hand, QP3A assigns the VLC APs to the Class 1

TABLE II

NORMALISED VLC AND MMW CHANNEL GAINS W.R.T THE USER POSITIONS CONSIDERED IN TABLE I (IN DBM)

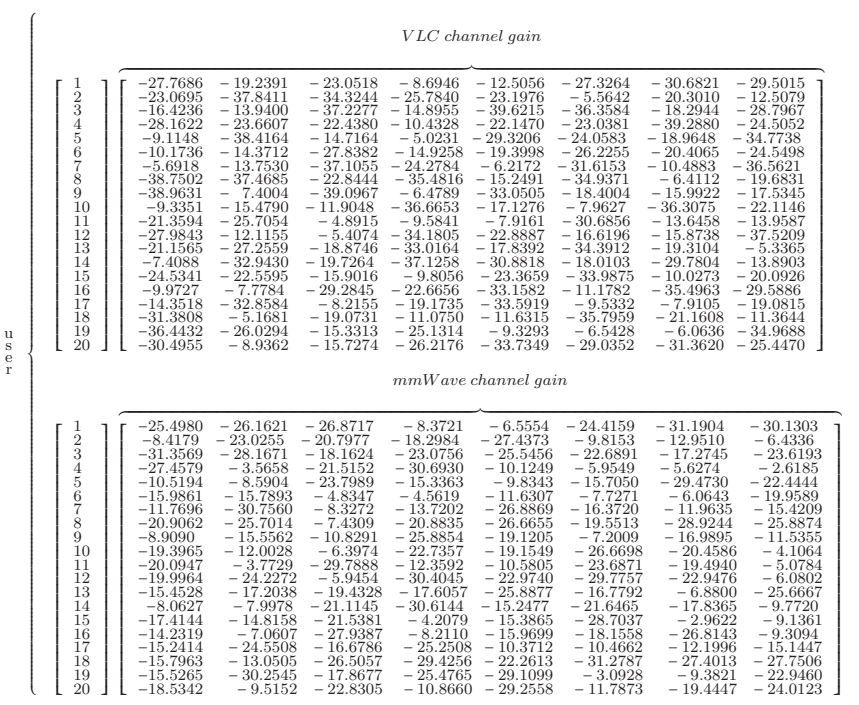

TABLE III

INDIVIDUAL USER PRIORITIES AND MINIMUM QOS REQUIREMENTS CONSIDERED FOR EVALUATION Conventional PO2A

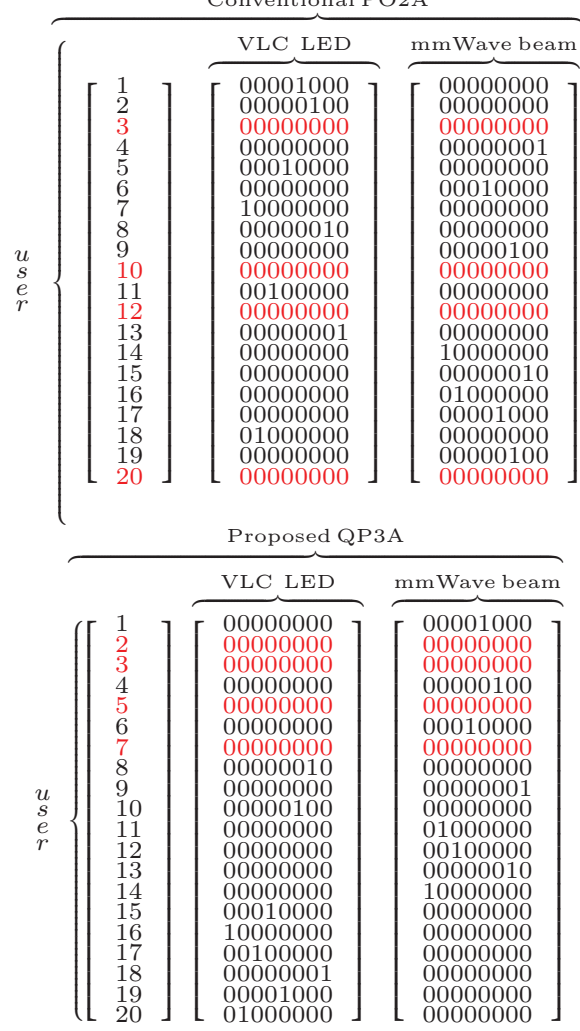

and Class 2 users, the mmWave APs to the Class 2 users and the residual mmWave APs to the Class 3 users. The QP3A allocation is fairer since only the Class 3 users are assigned to the WiFi network, which is capable enough to serve their minimum QoS requirements. However, as Class 3 users may have higher channel gains than Class 1 and Class 2 users, the QP3A overall throughput is likely to be slightly less than the overall PO2A throughput, which we examine via Matlab simulations in next Section.

\section{B. Evaluations using Matlab Simulink simulations}

Upon considering either the user priorities or the minimum QoS specified in Table I, we see at the left-hand side of Fig. 3 that the overall throughput of QP3A is $2.68 \mathrm{Gbps}$ in average, while upon considering both the individual user priorities and minimum QoS, it decreases to 2.58 Gbps in average. This is because at each time interval some Class 1 users and/or some users with high $\mathrm{QoS}_{j}^{\mathrm{min}}$ are placed in dead zones, where either the VLC or the mmWave or even both APs have particularly low SNR (i.e. LED illuminance and/or beam power density are low). However, although its decreased throughput performance, the priority and QoS consideration in QP3A enables it to decide the AP allocation at each time interval in a fairer manner compared to PO2A. This can be seen at the right-hand side of Fig. 3, where we plot the Jain's fairness index (FI) at each time interval defined as $F I=\left(\sum_{j=1}^{J} R_{j}\right)^{2} / J \cdot \sum_{j=1}^{J} R_{j}^{2}$, which is considered as convenient metric to rate the fairness over a set of given values [19]. From the graph we observe that all three versions of QP3A result to $F I>0.9$, which, in physical terms, indicates exceptional perception of our algorithm for accounting the individual user priorities and QoS demands. In contrast, PO2A 


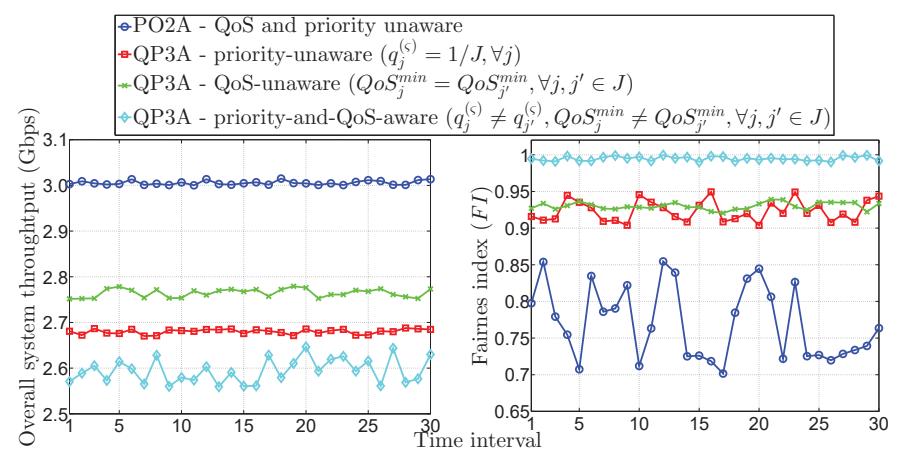

Fig. 3. Illustration of (i) the overall system throughput (left), and (ii) fairness index (right) versus the time intervals considering PO2A APA strategy and three versions of QP3A, i.e., priority-unaware, QoS-unaware and priority-andQoS-aware.

results to $0.7 \leq F I \leq 0.85$, which indicates that fairness occurs rather asymptotically than systematically as in QP3A. Consequently, QP3A facilitates a more convenient way for addressing the throughput-vs-fairness trade-off of the hybrid network than PO2A by allocating APs according to users' minimum QoS demands, priorities and positioning.

Furthermore, Fig. 4 examines the complexity of the algorithms at hand, considering that each iteration lasts 50 msec. As observed at the left-hand side of the figure, PO2A implements in about 10 times more iterations than QP3A. We also see by the right-hand side of the figure that the time required by $\mathrm{QP} 3 \mathrm{~A}$ to converge is less than $1 \mathrm{sec}$, while PO2A needs 6 to 9 secs. This confirms that the our solution method can resolve the 3D-APA problem particularly fast (i.e. close to real-time) because the optimal indexing in (14) and the feasibility criterion in (16) are both independent from the additional Lagrangian multipliers that are regularly involved during convex analysis. On the other hand, the PO2A-type algorithms in [2]-[11] and [13] are derived using traditional dual-Lagrangian solution method, which cannot get rid of the additional multipliers, thus, it requires more search processing to acquire the optimal points, which increases the computational time of those algorithms overall.

\section{CONCLUSION AND FUTURE WORK}

We modelled a hybrid system with three co-existing wireless access networks (VLC, mmWave and $\mathrm{WiFi}$ ) and formulated the corresponding 3D-APA problem towards maximising the overall network throughput subject to individual user positioning, QoS and priority constraints. We used time-sharing for the SNR to scale with the user indexing and relaxed the problem from NP-hard into convex, where we introduced a new method for deriving the optimal solution independently from the Lagrange multipliers, i.e., in linear polynomial time. We used the IoRL real-world SDN testbed and Matlab simulations to evaluate that the proposed solution prioritises allocating the most demanded and high-class users either to VLC or mmWave APs, which improves the throughput-vsfairness trade-off compared to conventional APA approaches, where some of these users are dropped, rather unfairly and suboptimaly, to the WiFi network. Our future efforts will focus on merging 3D-APA with load balancing schemes for calibrating AP assignments together with the over-load and under-load data traffic state of each of the co-existing networks in runtime.

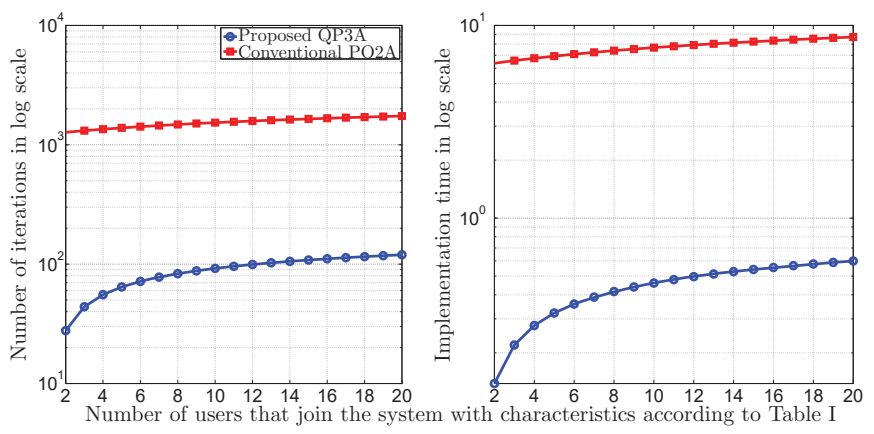

Fig. 4. Illustration of the implementation complexity between $\mathrm{PO} 2 \mathrm{~A}$ and QP3A: number of iterations and implementation time versus the number of users joining the system.

\section{REFERENCES}

[1] EU H2020 Internet of Radio Light (IoRL) project, [Online]: https://5gppp.eu/iorl/

[2] D. A. Basnayaka and H. Haas, "Hybrid RF and VLC systems: Improving user data rate performance of VLC systems," IEEE Veh. Technol. Conf., pp. 1-5, Glasgow, UK, May 2015

[3] X. Li, R. Zhang, and L. Hanzo, "Cooperative load balancing in hybrid visible light communications and WiFi," IEEE Trans. Commun., vol. 63, no. 4, pp. 1319-1329, 2015

[4] Y. Wang, D. A. Basnayaka, and H. Haas, "Dynamic load balancing for hybrid $\mathrm{Li}-\mathrm{Fi}$ and RF indoor networks," IEEE Int. Conf. Commun., pp. 1422-1427, London, UK, 2015

[5] F. Kelly, "Charging and rate control for elastic traffic," IEEE Trans. on Emerging Telecommun. Technol., vol. 8, no. 1, pp. 33-37, 1997

[6] Y. Wang and H. Haas, "Dynamic load balancing with handover in hybrid Li-Fi and Wi-Fi networks," IEEE/OSA J. Lightw. Technol., vol. 33, no. 22, pp. 4671-4682, Nov. 2015

[7] Y. Wang, D. A. Basnayaka, X. Wu, and H. Haas, "Optimization of load balancing in hybrid LiFi/RF networks," IEEE Trans. Commun., vol. 65, no. 4, pp. $1708-1720,2017$

[8] X. Wu, M. Safari, and H. Haas, "Joint optimisation of load balancing and handover for hybrid LiFi and WiFi networks," IEEE Wireless Commun. and Netw. Conf., pp. 1-5, San Francisco, US, 2017

[9] X. Wu and H. Haas, "Access point assignment in hybrid LiFi and WiFi networks in consideration of LiFi channel blockage," IEEE Workshop in Signal Processing Advances in Wireless Commun., 2017

[10] X. Wu, D. Basnayaka, M. Safari, and H. Haas, "Two-stage access point selection for hybrid VLC and RF networks," IEEE Conf. Personal, Indoor, and Mobile Radio Commun., pp. 1-6, 2016

[11] F. Kelly, "Charging and rate control for elastic traffic," Transactions on Emerging Telecommunications Technologies, vol. 8, no. 1, pp. 33-37, 1997

[12] Y. Wang and H. Haas, "A comparison of load balancing techniques for hybrid LiFi/RF networks," ACM Workshop on Visible Light Commun. Systems, pp. 43-47, 2017

[13] H. Zhang, N. Liu, K. Long, J. Cheng, V. C. M. Leung, and L. Hanzo, "Energy efficient subchannel and power allocation for the software defined heterogeneous VLC and RF networks," IEEE J. Selected Areas Commun., vol. 1 no. 99 , pp. 1-1, 2017

[14] S. Boyd and L. Vandenberghe, "Convex Optimization", Cambridge University Press, 2009

[15] EU H2020 Internet of Radio Light (IoRL) project, Deliverable 3.2, [Online]: https://iorl.5g-ppp.eu/deliverables/

[16] C. C. Zarakovitis and Q. Ni, "Maximizing Energy Efficiency in Multiuser Multicarrier Broadband Wireless Systems: Convex Relaxation and Global Optimization Techniques," IEEE Trans. Veh. Technol, vol. 65, no. 7, pp. 5275-5286, Jul 2016

[17] R. M. Corless, G. H. Gonnet, D. E. G. Hare, D. J. Jeffrey, and D. E. Knuth, "On the Lambert W Function," Adv. Compu.Math, pp. 329-359, 1999

[18] Ryu SDN controller, [Online]: https://osrg.github.io/ryu/

[19] Jain's fairness index [Online]: https://en.wikipedia.org/wiki/Fairness_measure

[20] H. Zhang, N. Liu, K. Long, J. Cheng, V. C. M. Leung, and L. Hanzo, "Energy efficient subchannel and power allocation for the software defined heterogeneous VLC and RF networks," IEEE J. Sel. Areas Commun., vol. 36, no. 3, pp. 658-670, Mar 2018 